\title{
Mineral weathering and nutrient cycling at the early stages of soil development
}

\author{
CELIA ARANDA REINA ${ }^{1}$, BASTIEN WILD ${ }^{1}$, JORDI \\ CAMA $^{2}$ AND IAN BOURG ${ }^{1}$
}

${ }^{1}$ Princeton University

${ }^{2}$ Institute of Environmental Assessment and Water Research (IDAEA-CSIC)

Presenting Author: creina@alumni.princeton.edu

Mineral weathering is key to the long-term supply of ecosystems with bioavailable nutrients, and microbial communities play a central role in the uptake and transfer of nutrients. These processes are of particular significance in contexts where recycling of biomass and/or atmospheric input may not be sufficient to support biomass buildup or to compensate the depletion of soil nutrient pools associated with human activities. In this study, we examined the potential for biotically and abiotically-controlled weathering of minerals in the forefield of a receding glacier located in the Spanish Pyrenees, representative of early stages of soil development. We performed laboratory dissolution experiments, some of which contained soil samples endowed with their native microbial communities. Dissolution rates of a selection of silicate, carbonate, and phosphate minerals was determined by combining fluid concentration analyses with mineral surface topography measurements by atomic force microscopy (AFM). We investigated the detail of mineral surface dynamics, including etch pits formation, in each condition using AFM, while the evolution of microbial communities was evaluated based on bacterial 16S rRNA gene survey. Our experiments unraveled the relative role of surface dynamics, fluid saturation and soil microorganisms on mineral dissolution rates. Our results, and the associated methodological developments, pave the way towards a planned in-situ characterization of mineral weathering rates in the critical zone. 